\title{
Recent developments in advanced diagnostic bronchoscopy
}

\author{
Sharad Chandrika and Lonny Yarmus \\ Number 2 in the Series "Interventional pulmonology" \\ Edited by David Feller-Kopman and Hervé Dutau
}

Affiliation: Division of Pulmonary and Critical Care Medicine, Johns Hopkins University, Baltimore, MD, USA.

Correspondence: Sharad Chandrika, Division of Pulmonary Critical Care Medicine, Johns Hopkins University, 1800 Orleans Street, Baltimore, MD, 21218-2625, USA. E-mail: sharad.chandrikadaymail.com

@ERSpublications

Advanced diagnostic bronchoscopy is a brilliant example of interdisciplinary collaboration. Making the best of these newly available tools will be guided by the proceduralists' experience, the patient and target lesion characteristics. https://bit.ly/33Y0TQsppl

Cite this article as: Chandrika S, Yarmus L. Recent developments in advanced diagnostic bronchoscopy. Eur Respir Rev 2020; 29: 190184 [https://doi.org/10.1183/16000617.0184-2019].

ABSTRACT The field of bronchoscopy is advancing rapidly. Minimally invasive diagnostic approaches are replacing more aggressive surgical ones for the diagnosis and staging of lung cancer. Evolving diagnostic modalities allow early detection and serve as an adjunct to early treatment, ideally influencing patient outcomes. In this review, we will elaborate on recent bronchoscopic developments as well as some promising investigational tools and approaches in development. We aim to offer a concise overview of the significant advances in the field of advanced bronchoscopy and to put them into clinical context. We will also address potential complications and current diagnostic challenges associated with sampling central and peripheral lung lesions.

\section{Introduction}

Gustav Killian, the "father of bronchoscopy", performed the first bronchoscopy in 1876 when he removed a pork bone from a farmer's airway, forever altering the landscape of pulmonology [1, 2]. Almost a century later, Shigeto Ikeda invented the fibreoptic bronchoscope revolutionising the field of bronchoscopy. Since then, the development of modern advanced diagnostic bronchoscopy has spawned more than 50 years of innovation and exploration [1]. A significant turning point in the history of modern diagnostic bronchoscopy occurred with the invention of endobronchial ultrasound-guided transbronchial needle aspiration (EBUS-TBNA). The widespread adoption and guideline integration of EBUS-TBNA led to a paradigm shift in the minimally invasive care of the lung cancer patient.

Advanced diagnostic bronchoscopy has had exponential growth over the past decade. This evolution occurred on a backdrop of extraordinary progress in the field of thoracic oncology. With the advent of personalised medicine, the outcomes and survival of patients with lung cancer are better than ever, necessitating the need to predict the precise molecular makeup of their tumour environment. Improvements in sedation techniques and radiographic tools have also further assisted advancements in

Previous articles in this series: No. 1: DeMaio A, Sterman D. Bronchoscopic intratumoral therapies for nonsmall cell lung cancer. Eur Respir Rev 2020; 29: 200028.

Provenance: Commissioned article, peer reviewed

Received: 19 Dec 2019 | Accepted after revision: 24 March 2020

Copyright (CERS 2020. This article is open access and distributed under the terms of the Creative Commons Attribution Non-Commercial Licence 4.0. 
bronchoscopic technology, which provides a blueprint based on which tissue acquisition site and location is selected.

In this article, we will be describing a myriad of innovations that have occurred over the past decade that will prove to be stepping stones to bigger milestones in the journey of advanced diagnostic bronchoscopy.

\section{Mediastinal lesions}

\section{Convex probe-endobronchial ultrasound}

The first case of sampling a mediastinal tumour via flexible bronchoscopy was reported by Ko-Pen Wang in 1978 [3]. Conventional Transbronchial Needle Aspiration (C-TBNA) revolutionised the access to mediastinal and hilar masses, later paving the way for the advent of endobronchial ultrasound (EBUS). Although transbronchial needle aspiration (TBNA) was a major landmark in mediastinal/hilar sampling, it suffered from limited adoption due to limitations in training technique, variable yields and conditioned concerns for safety $[4,5]$.

Many of the concerns regarding the use and adoption of C-TBNA were allayed with the introduction of EBUS-TBNA. The first report on the clinical application of linear probe EBUS was published in 2004 [6]. The literature from the ASTER trial in 2013 integrated the era of EBUS-TBNA in the staging and diagnostic algorithm of cancer in the lung and mediastinum [7].

EBUS-TBNA uses ultrasound technology via a probe at the distal tip of the bronchoscope to perform TBNA of the hilar and mediastinal lesions under direct visualisation with ultrasonic images [8]. Figure 1 illustrates a hybrid-EBUS bronchoscope and high-definition images of the airway.

EBUS-TBNA has been compared with mediastinoscopy for mediastinal lymph node staging of potentially resectable nonsmall cell lung cancer with $91 \%$ agreement shown between both techniques. Specificity and positive predictive value for both techniques were $100 \%$ [8]. Convex probe (CP)-endobronchial ultrasound has largely replaced conventional TBNA and has taken over from mediastinoscopy as the "gold standard" for lung cancer staging [9].

The American College of Chest Physicians (ACCP) guidelines offering evidence-based recommendations on technical aspects of the procedure proclaims EBUS-TBNA to be the test of choice for the initial staging of lung cancer patients [10,11]. The bronchoscopic approach of EBUS-TBNA also has an integrated benefit of providing diagnosis and staging at the same time to ideally avoid putting patients through the added risks of additional invasive procedures such as transbronchial biopsy (TBB) or computed tomography (CT)-guided transthoracic needle aspiration. The latter, if done first, may also require an additional invasive procedure to stage the disease $[8,12]$.

EBUS-TBNA has reported pooled sensitivity of roughly $90 \%$ for the staging of lung cancer $[6,13]$. These statistics are comparable to a pooled sensitivity of $89 \%$ for video mediastinoscopy, which is considered superior to that of conventional cervical mediastinoscopy $[7,10]$. EBUS has a reported negative predictive value (NPV) of $92 \%$ for occult metastatic disease (i.e. in cases of pathological N2 disease but radiographic $\mathrm{N} 0$ or N1 disease based on CT and/or positron emission tomography (PET)) [8].

A study of 981 patients from 2009 to 2014 analysed the role of preoperative EBUS-TBNA in patients with lung cancer without involvement of the mediastinum at PET-CT. Of the 167 patients included, 6.0\% were upstaged to N2 or N3 by EBUS-TBNA; nine of these were originally classified as N1 at PET-CT. Thereby $17.3 \%$ of the included $\mathrm{N} 1$ patients were upstaged to N2/N3 with EBUS-TBNA, hence proving the overall probability of a clinically relevant upstaging by EBUS-TBNA in patients initially deemed N0/N1 at PET-CT as $6.0 \%$, compared to $0.9 \%$ in patients classified as N0 and $17.3 \%$ in patients classified as N1 [14].

In the era of personalised medicine, the demand for molecular tumour profiling of a patient's tumour is increasing, which is based on the idea that tumour-specific biomarkers predict patient's prognosis and tumour response to therapy. This, in turn, dictates the need for adequate and representative tissue specimens from recurrent or metastatic tumours or from patients who might have developed resistance to initial therapies. EBUS provides a convenient, relatively noninvasive means to resample the lesions with ease and high success rates with molecular yields of $>95 \%$ reported $[4,15,16]$.

The yield of EBUS for diagnosing and subtyping lymphoma (particularly de novo lymphoma) remains lower at $\sim 70 \%$, with a considerable variation in estimates. Mediastinoscopy is still considered the test of choice if lymphoma is high on the differential diagnosis $[8,12]$.

In the right clinical context, EBUS-TBNA also demonstrates excellent diagnostic accuracy for infectious aetiologies such as mediastinal tuberculosis (TB) lymphadenitis. A multicentre retrospective study analysed 159 patients that underwent EBUS-TBNA and had tissue referred for mycobacterial culture; 39 (25\%) were ultimately diagnosed with TB. Reported sensitivity and specificity of EBUS-TBNA for microbiologically 

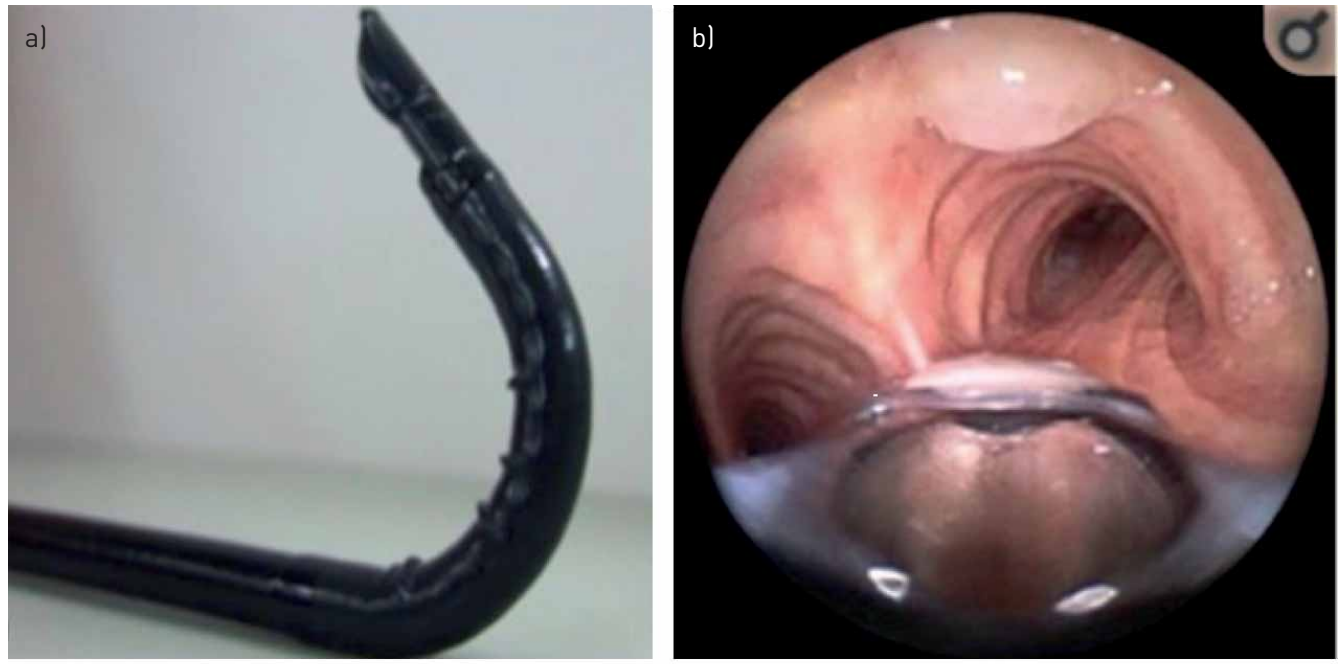

c)

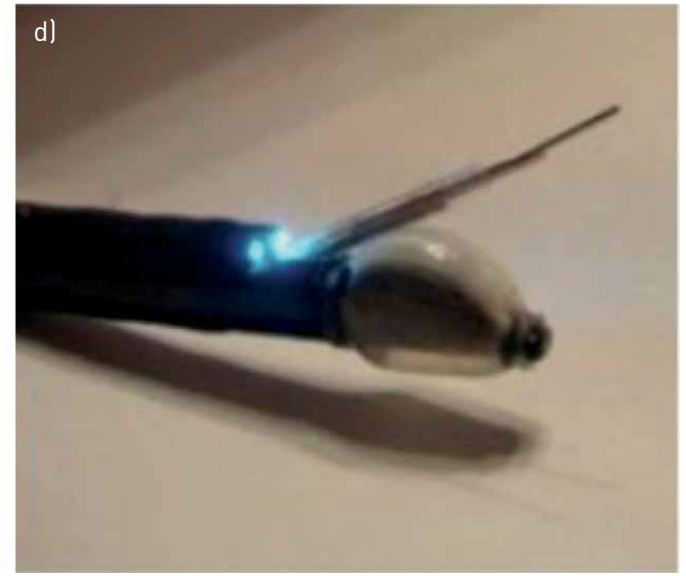

FIGURE 1 Hybrid endobronchial ultrasound bronchoscope and high-definition image of the airway. a) Tip of the flexed endobronchial ultrasound bronchoscope. b) Endobronchial view. c) Convex area of the scope. d) Needle extending through the work channel.

confirmed tuberculous mediastinal lymphadenitis were 62\% (24 out of 39 cases) and 100\%, respectively. NPV and diagnostic accuracy for microbiological diagnosis were $89 \%$ and $91 \%$, respectively. For a composite clinicopathological diagnosis of TB, NPV and accuracy were 98\% and 98\% (95\% CI 95-99\%), respectively [17].

EBUS has proven utility in the evaluation of sarcoidosis. A prospective study of 62 patients with suspected stage I and II sarcoidosis where EBUS-TBNA was performed, followed by transbronchial lung biopsy (TBLB) in the same setting, reported diagnostic yield of EBUS-TBNA and TBLB for sarcoidosis by showing noncaseating epithelioid cell granuloma was 94\% (stage I: 97\%; stage II: 88\%) and 37\% (stage I: 31\%; stage II: 50\%), respectively [18]. Another study reported $85 \%$ sensitivity of EBUS-TBNA for pulmonary sarcoidosis. By combining multiple modalities like conventional transbronchial biopsies, endobronchial mucosal biopsy and EBUS, yield could be further enhanced [11].

CP-EBUS also has applications beyond conventional indications, such as cyst drainage and therapeutic drug injections [19].

\section{Concurrent EBUS and endoscopic ultrasound}

Ample data indicate that concurrent EBUS and endoscopic ultrasound (EUS) increases diagnostic yield by a statistically significant margin. A needle-based technique (either EBUS-TBNA, EUS-TBNA or a combination) has been recommended as the first line for mediastinal nodal staging of known or suspected lung cancer [10]. The combination of EUS and EBUS is preferred over either test alone for mediastinal nodal staging. EBUS alone is considered acceptable if the combination of EBUS and EUS is not available [20].

The use of combined EBUS-TBNA and EUS-guided fine-needle aspiration (FNA) for preoperative hilar and mediastinal staging of nonsmall cell lung cancer (NSCLC) with a single bronchoscope in a single 
session has been elucidated in detail. In a prospective study of 150 patients with potentially resectable known or suspected NSCLC, reported sensitivities of EBUS, EUS and the combined approach per patient were $52 \%, 45 \%$ and $73 \%$, respectively. The use of a combined endoscopic approach with EBUS-TBNA and EUS-FNA was shown to be a safer and more precise method for preoperative hilar and mediastinal staging of NSCLC, with better reported results than with each technique by itself [21].

EBUS is limited to the anterosuperior mediastinum, and EUS is utilised to sample the posteroinferior mediastinum, which justifies the case for combining EBUS with EUS for a more thorough and systematic mediastinal staging [22].

\section{Challenges of EBUS technology}

Sampling with a needle-based technology is dependent upon availability of the technology, expertise of the operator and resources for adequate specimen interpretation [11]. Cost of acquiring EBUS/EUS technologies is another limiting factor; however, the procedure itself may be cost-effective when other factors such as avoidance of other invasive surgical procedures are weighed up [23].

Another limitation is the relative complexity of the handling of the EBUS needle that involves several steps. The EBUS procedure usually requires general anaesthesia or deep sedation with laryngeal mask airway or an endotracheal tube in order to have a satisfying operative process.

\section{Future implications}

Higher resolution and smaller size scopes, compounded with other technologies, such as three-dimensional ultrasound, ultrasonic contrast and harmonic imaging, incorporated to further enhance the diagnostic ability of EBUS are currently being evaluated [19].

\section{Peripheral lesions}

Demonstration of a significant number of nodules in lung cancer screening trials [24, 25] coupled with the numerous limitations of conventional bronchoscopic techniques have driven the massive efforts of finding complementary bronchoscopic techniques such as virtual bronchoscopy, electromagnetic navigation bronchoscopy (ENB), radial endobronchial ultrasound (r-EBUS), robotic bronchoscopy and hybrid modalities. Diagnostic bronchoscopy has evolved with the availability of a vast arsenal of sophisticated tools. Making the best of these tools will mainly be guided by the proceduralist's experience, the patient and tumour characteristics.

\section{$r$-EBUS}

While central airway radial ultrasound probes are used to detail imaging of the airway wall and surrounding structures, peripheral radial probes allow for visualisation and subsequent sampling of peripheral intrapulmonary lesions [26]. Two types of EBUS radial probes are commercially available in the USA. The $20 \mathrm{MHz}$ radial probe EBUS fitted with a catheter that has a water-inflatable balloon at the tip is used to evaluate central airways (trachea/subsegmental bronchus). The $20 \mathrm{MHz}$ ultra-miniature radial probe can be inserted through a $2 \mathrm{~mm}$ working channel of a diagnostic bronchoscope and can be extended into subsegmental bronchi housed in a guide sheath [27]. The guide sheath-covered probe is advanced to the peripheral lesion (could be used with the fluoroscopy) to obtain an EBUS view. Once the lesion is localised, the probe is removed, leaving the guide sheath in place. A biopsy instrument (forceps, needle, bronchial brush) is then inserted through the guide sheath to obtain specimens [28].

r-EBUS, initially described in 1992, was utilised to guide TBNA and to assess tumour invasion versus compression in the more central larger airways [29]. With the advent of CP-EBUS allowing for real-time visualisation during biopsy of central lesions, use of r-EBUS is now mainly confined to intraprocedural imaging of peripheral lesions, which is performed using the peripheral radial probe [30]. A specificity of $100 \%$ and sensitivity of $73 \%$ for lung cancer detection were reported in a 2011 meta-analysis (16 trials with 1420 patients) that employed bronchoscopy with r-EBUS-assisted peripheral nodule for sampling [31]. The diagnostic yield with r-EBUS increases with the presence of a CT bronchus sign [32]. A recent study of 147 patients showed that diagnostic yield with r-EBUS was only significantly influenced by visualisation with the r-EBUS (OR 3.70 (95\% CI 1.35-11.02), $\mathrm{p}=0.014$ ). Also, location ( $\mathrm{p}=0.745$ ) and size $>30 \mathrm{~mm}(\mathrm{p}=0.308)$ showed no significant increase in diagnostic yield [33].

However, a recent prospective multicentre randomised trial compared the diagnostic yield of a thin bronchoscope and r-EBUS with standard bronchoscopy and fluoroscopy in peripheral pulmonary lesions [34]. They reported a somewhat higher diagnostic yield in the r-EBUS arm compared with the standard bronchoscopy and fluoroscopy arm (49\% versus 37\%); the difference, however, was not statistically significant. The study above concluded that bronchoscopy with or without a thin scope and r-EBUS had a poor diagnostic yield for pulmonary lesions. 
A recent feasibility study for the sampling of peripheral pulmonary lesions tested a prototype of a real-time visualisation device integrating r-EBUS and biopsy needle into a single device (figure 2) that can be passed through a 2.0-mm working channel to sample lesions in real-time similar to EBUS-TBNA [35]. This promising innovation may shape peripheral sampling in a whole new way by improving the efficiency of tissue sampling. An r-EBUS biopsy tool is shown in figure 3.

\section{ENB and virtual bronchoscopic navigation}

Navigation bronchoscopy, as the name implies, uses a navigational system to guide instruments (e.g. flexible or ultra-thin bronchoscope) through the airways to sample a target lesion. Navigational systems can be virtual (virtual bronchoscopic navigation, usually non-contrast enhanced CT) or electromagnetic (ENB).

Virtual bronchoscopic navigation encompasses multi-row detector CT-derived images, other computer graphics and computer vision-derived value-added digital imagery to create three-dimensional image renderings of the bronchial tree $[36,37]$. Software solutions can then produce simple renderings of the bronchial tree through which a fly-through of the airway lumen can be reproduced, thereby mimicking the view of a real flexible bronchoscope $[38,39]$. The three-dimensional image of the thorax obtained through multi-row detector CT is a digital image referred to as a "voxel", with each voxel having a defined spatial dimension and grey-scale characteristics, but virtual bronchoscopy does not provide real-time positional guidance [39].

In contrast, electromagnetic navigation (EMN) bronchoscopy relies on a pre-procedural CT of the chest to create a three-dimensional virtual airway map, which is then linked to an electromagnetic field to provide spatial feedback in real-time [40]. The ACCP recommends that in patients with peripheral lung lesions that are difficult to reach with conventional bronchoscopy, electromagnetic guidance be performed if the equipment and expertise are available [41]. The bronchoscopic procedure may or may not be aided with fluoroscopic guidance. ENB has been found to be complementary to r-EBUS [41].

Various navigation technologies, including virtual bronchoscopy and real-time tracking using EMN platforms, are commercially available. Each one of the commercially available systems SuperDimension (Medtronic, Dublin, Ireland), LungPoint (Broncus Medical, Inc., San Jose, CA, USA) and the SPiN System (Veran Medical Technologies, St. Louis, MO, USA) employs slightly different technologies for access and sampling. LungPoint relies solely on virtual bronchoscopy without the added EMN component. LungPoint is the only system that provides "bronchoscopic transbronchial parenchymal nodule access" (i.e. direct tunnelling through normal parenchyma), although there are limited data showing efficacy [42].

Another novel technology, electromagnetic guidance transthoracic needle aspiration (ETTNA), incorporates a unique electromagnetic guidance system that allows clinicians to track solitary pulmonary nodule (SPN) and target them for ETTNA without utilising real-time CT in the operating room or bronchoscopy suite [43]. In a single-centre, prospective pilot study examining the safety, feasibility and

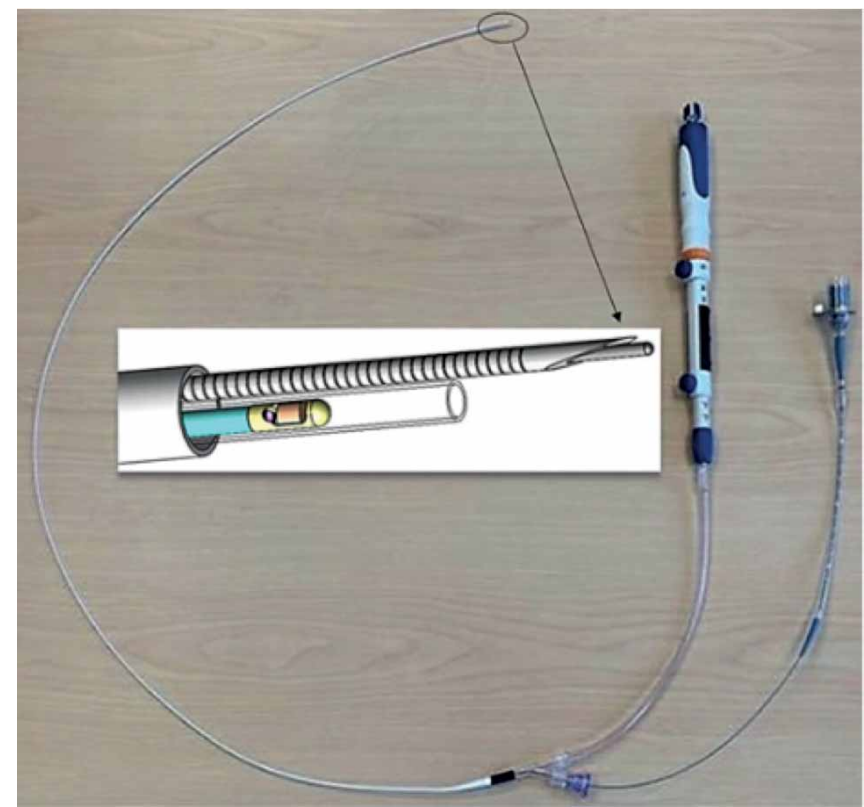




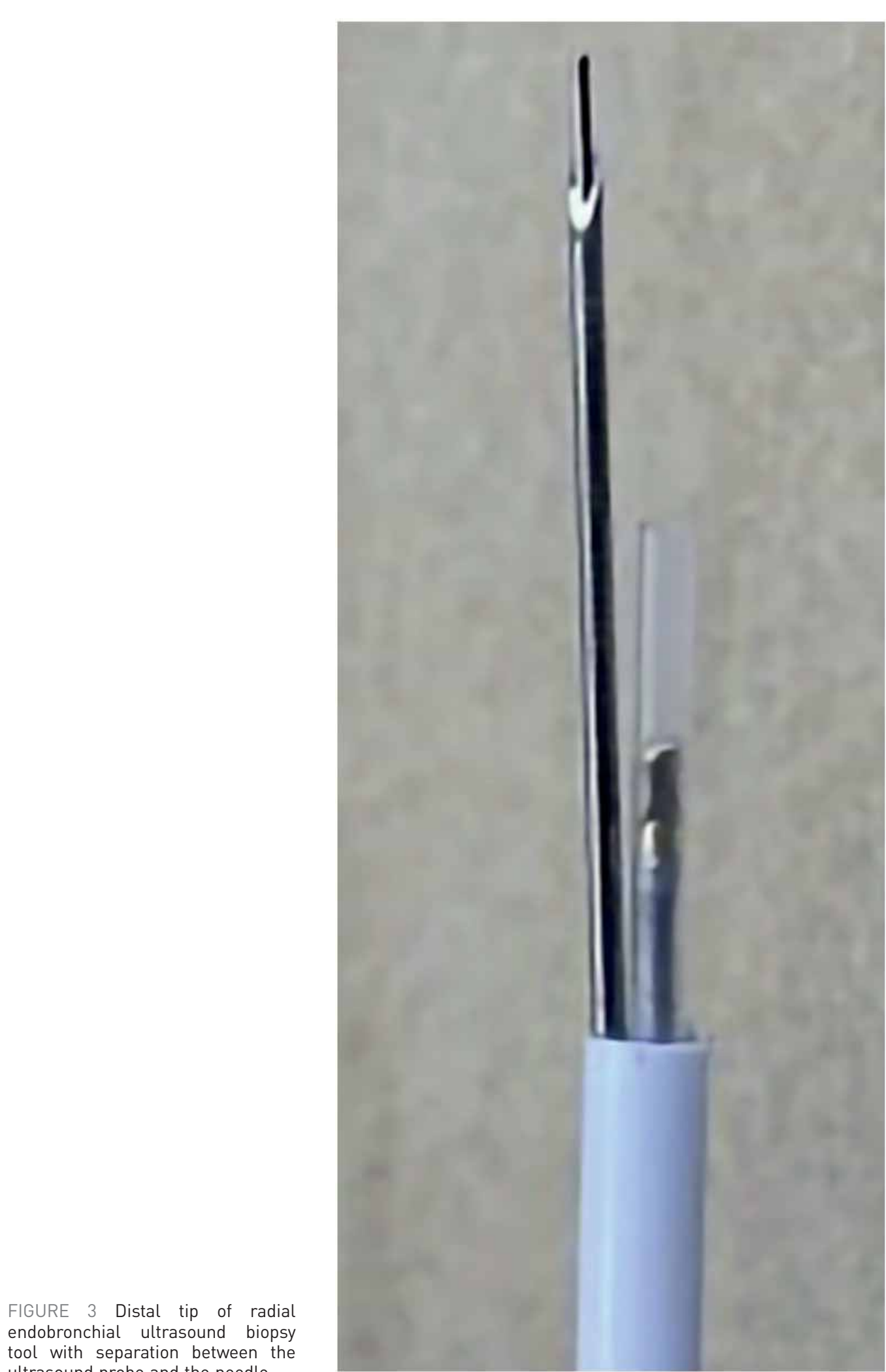
ultrasound probe and the needle.

diagnostic yield of combined ENB and ETTNA, diagnostic yield for ETTNA alone was reported to be $83 \%$ and further increased to $87 \%$ when combined with ENB. The diagnostic yield increased to $92 \%$ when all three modalities were combined [44]. A large multicentre prospective clinical trial validating this technique completed enrolment in 2019, and results are expected in 2020 [45]. 


\section{Challenges of navigational bronchoscopy}

Results of the AQuIRE registry challenged the role of navigational bronchoscopy for peripheral pulmonary lesions with the reported yield for EMN being only 39\%. The factor most amenable to physician control that improved diagnostic yield was surprisingly the use of peripheral TBNA [46]. Respiratory motion of the airways, lung, and nodule hysteresis creates unique challenges for targeting the lesion. Navigation errors and the inability of navigation to directly confirm the target prior to biopsy also inversely affect the yield. Concurrent use of fluoroscopy and r-EBUS may provide meaningful information and additional confirmation with difficult lesions. The virtual nature of these bronchoscopic modalities may explain the variability in diagnostic rates. Additional studies are needed to determine if these factors can be overcome to improve yield.

\section{CT bronchoscopy/image-guided bronchoscopy/augmented fluoroscopy}

Fluoroscopy is the current guidance tool for TBB, which is limited by two-dimensional imaging and associated significant cumulative radiation dose for the patient and the operator.

Consecutive problem-based solutions and refinements are urgent requisites to achieve improvements in yield. The technique of "augmented fluoroscopy" creates an overlay of the target on live fluoroscopic images hence the term "augmented". Augmented fluoroscopy has limited data, and thus its true utility remains unknown, but a prospective randomised trial is in the final planning stages [47].

Cone-beam CT allows real-time visualisation of the target lesion (figure 4) while the sampling tool is still in place, which may overcome the limitations of other modalities, but there are limited studies to date.

A prospective randomised trial comparing CT fluoroscopy-guided bronchoscopy with conventional bronchoscopy for lung cancer diagnosis failed to demonstrate a significant difference between either modality both in peripheral lesions and mediastinal lymph nodes [48]. The diagnostic accuracy of CT was higher for confirmation of target entry. There was a trend suggesting the superiority of CT scan guidance on a per-lymph-node entry basis.

Despite the feasibility of cone-beam CT-derived three-dimensional road mapping navigation for bronchoscopic procedures using augmented fluoroscopy, its impact upon workflow, outcomes and cost are undefined as are the potential roles for these tools in clinical care [49]. Before there is any meaningful adoption of these expensive tools, studies that accurately assess yield and cost effectiveness are needed.

\section{Robotic bronchoscopy}

The diagnostic accuracy of currently available bronchoscopic tools remains limited, invoking the dire necessity for more innovative devices. Hence the concept of robotic bronchoscopy emerged with the hope of overcoming the limitations of other image-guided sampling techniques. The first robotic bronchoscopic system received US Food and Drug Administration approval for commercial use in 2018, soon to be followed by a second manufacturer in early 2019 [50]. A recent pilot study on 15 patients demonstrated the safety and feasibility of robotic bronchoscopy [51].

a)

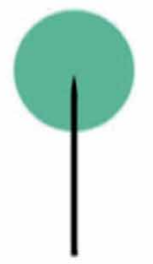

Centre

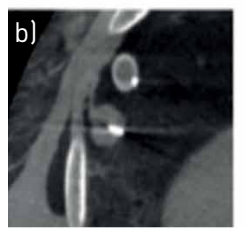

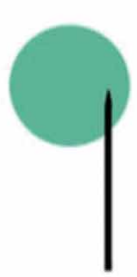

Peripheral

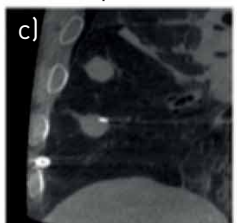

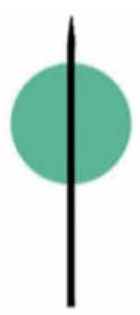

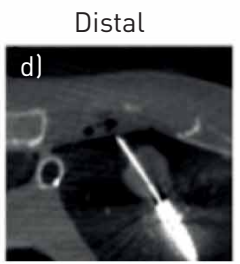

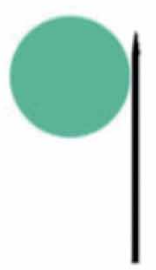

Adjacent

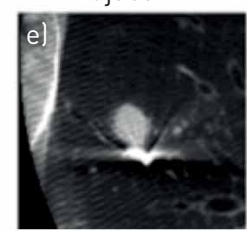

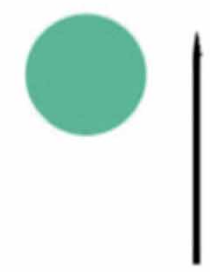

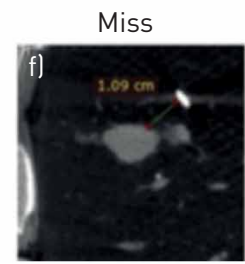

FIGURE 4 a) Illustration of various possible needle puncture scenarios seen on cone-beam computed tomography imaging. Needle b) entering in the center of the lesion, c) from the periphery, d) going too distally through the lesion, el just adjacent to the lesion, and f) missing the lesion completely. 
The system combines a robotically controlled catheter, with direct airway visualisation, navigated through the airways along a virtual pathway to a target nodule. The robotic components allow controlled advancement of the catheter as well as subtle directional movements in all planes at the catheter tip. The catheter has the unique property of maintaining a single position and angulation while not being advanced, with a sufficiently large working channel $(2 \mathrm{~mm})$ to allow passage of biopsy instruments [52, 53].

A safety and feasibility study in patients with small peripheral lesions measuring between 1 and $3 \mathrm{~cm}$ showed an overall diagnostic yield of $83 \%$, and diagnostic yield for malignancy was $89 \%$ [52]. No device-related adverse events occurred; in particular, no instances of pneumothorax or excessive bleeding were observed.

A recent randomised controlled comparative study in a human cadaver model showed that the utilisation of robotic bronchoscopy significantly increased the ability to localise and successfully puncture small peripheral pulmonary nodule when compared with existing technologies including ultra-thin bronchoscope with radial EBUS versus EMN [54]. Multicentre studies comparing other image-guided modalities are required to assess its performance.

\section{Parenchymal lung disease \\ Cryobiopsy}

Transbronchial cryobiopsy (TBCB) is gaining popularity in the diagnostic approach to diffuse parenchymal lung diseases. TBCB is the technique of performing TBLB via bronchoscopic placement of a flexible cryoprobe inside the lung parenchyma, freezing the probe and shearing out the frozen lung tissue. Cryobiopsy has been recognised to provide larger specimens than the conventional lung biopsies, more alveolar tissue, minimal crush artefact and better diagnostic yield compared to conventional TBB. Despite the larger specimen size and the promise of tissue preservation, several concerns persist about the optimal procedural technique and safety profile.

In 2018 an expert statement from the Cryobiopsy Working Group formulated evidence- and expert-based suggestions on the procedural aspects of the TBCB procedure [55]. It was suggested that TBCBs be performed by interventional bronchoscopists trained at a centre with experience in TBCBs in the management of potential complications like bleeding, pneumothorax or respiratory failure [55]. The working group emphasised that biopsies with distances of $<1 \mathrm{~cm}$ from the pleura may be associated with a significantly higher risk of pneumothorax [56]. On the contrary, biopsies obtained too proximally from the middle third of the lung increase the risk of severe bleeding [57]. Therefore, optimal positioning of the cryoprobe with a distance of $1 \mathrm{~cm}$ from the pleura is recommended to minimise complications [56].

It was also suggested that a prospective registry be developed and all TBCBs recorded to capture morbidity and mortality data better [55].

A meta-analysis of 27 studies demonstrated a diagnostic yield of $72.9 \%$, together with a lower complication rate than surgical lung biopsy (SLB). A prospective, multicentre, comparative study conducted across nine Australian tertiary hospitals investigated diagnostic agreement between transbronchial lung cryobiopsy (TBLC) and SLB. The results showed 70.8\% (weighted $\kappa 0.70$, 95\% CI 0.55-0.86) histopathological agreement between TBLC and SLB with diagnostic agreement of $76.9 \%(\kappa 0.62,0.47-0.78)$ at multidisciplinary decisions. For TBLC with high or definite diagnostic concordance (39 (60\%) of 65 cases), 37 (95\%) were concordant with SLB diagnoses [56].

Despite an expanding body of literature, the technique is not yet standardised, and its place in the diagnostic algorithm of diffuse parenchymal lung diseases is not yet clearly defined. The optimal place for cryobiopsy in interstitial lung disease continues to be debated. Recently published ACCP guidelines on cryobiopsy for the diagnosis of interstitial lung disease is an important international and society-based effort to assist with optimising safety and yield while we await further studies.

\section{Confocal laser endomicroscopy}

Probe-based confocal laser endomicroscopy (pCLE) is an emerging high-resolution imaging technology that complements standard white-light bronchoscopy to provide in vivo and real-time imaging of the lungs [58]. A Japanese study explored the role of probe-based confocal laser endomicroscopy with acriflavine staining for rapid on-site evaluation of TBB specimens (sensitivity for malignancy was 91.3\%, specificity was 76.9\%) [59]. Feasibility and utility of pCLE in vivo microimaging of SPN has been described [60]. A study of 48 patients with malignant SPN demonstrated that pCLE imaging of SPNs is achievable in all of the segments of both lungs using either the $0.6-\mathrm{mm}$ or $1.4-\mathrm{mm}$ mini probe [61]. Future prospective studies, including comparison and combination with other modalities, are required to predict the presence of malignancy with high accuracy using standardised, reproducible descriptors. Until then, the use of this technology should be limited primarily to the research arena. 


\section{Novel insights}

\section{Elastography}

Elastography is a new ultrasonography-associated technology for the differentiation of benign and malignant lymph nodes before aspiration, which can be integrated with CP-EBUS.

In general, malignancy can render the tissues less deformable or hard. Elastography can be considered the imaging equivalent of palpation and therefore is a surrogate real-time method for the evaluation of tissue stiffness. These findings could be of potential value to better select targets for sampling during EBUS-FNA, thereby possibly reducing unnecessary needle passes and procedural time [62]. Limitations of elastography include variability of the elastographic images, the difficulty of interpretation, lack of clear definitions of the criteria required for an accurate elastographic evaluation and the endosonographers' inability to control tissue compression [63]. Elastography lacks standardisation as of now and further studies are needed.

\section{Optical coherence tomography}

Optical imaging and optical spectroscopy are steadily evolving as contenders for relatively noninvasive optical biopsy. Optical coherence tomography (OCT) is a time-of-flight imaging technology wherein images are generated by measuring the intensity of backscattered near-infrared light using low-coherence interferometry. OCT has found its promise in investigating airway-based lesions, including dysplasia, carcinoma in situ and invasive cancer [64, 65]. Needle-based OCT catheters can facilitate imaging beyond the limits of the airway wall $[66,67]$. Structural and polarisation-sensitive OCT, when used in conjunction, offer a synergistic depiction of both tissue microstructure and composition that may be useful in guiding biopsy site selection and hence optimising tumour content for both diagnosis and molecular profiling [68]. As of now, a lack of clinical studies and documentation of the image and spectral classification database limits its potential clinical use.

\section{Looking to the future}

The progress made by the advanced diagnostic bronchoscopy community since its introduction rivals that of other established major medical disciplines. With massive leaps taken, this ever-changing field is at the forefront of patient care, via continuous new clinical evidence. Interventional pulmonology is a brilliant example of cross-disciplinary collaboration. We anticipate continued innovation and hope for outstanding patient outcomes through this broad association.

Conflict of interest: S. Chandrika has nothing to disclose. L. Yarmus has received research grants and consulting fees from Veran medical, Boston Scientific, Intuitive, Olympus and Rocket Medical, outside the submitted work.

\section{References}

1 Panchabhai TS, Mehta AC. Historical perspectives of bronchoscopy: connecting the dots. Ann Am Thorac Soc 2015; 12: 631-641

2 Vaidya PJ, Leuppi JD, Chhajed PN. The evolution of flexible bronchoscopy: from historical luxury to utter necessity. Lung India 2015; 32: 208-210.

3 Yang $\mathrm{H}$, Zhang Y, Wang K-P, et al. Transbronchial needle aspiration: development history, current status and future perspective. $J$ Thorac Dis 2015; 7: Suppl. 4, S279-S286.

$4 \quad$ Xia Y, Wang K. Transbronchial needle aspiration: where are we now? J Thorac Dis 2013; 5: 678-682.

5 Zaman MK, Shrestha R. Major complications associated with conventional transbronchial needle aspiration. South Med J 2018; 111: 565-571.

6 Vilmann P, Larsen S, Krasnik M. EUS guided FNA for mediastinal tumors (lung cancer and lymph nodes). Dig Endosc 2004; 16: S185.

7 Rintoul RC, Glover MJ, Jackson C, et al. Cost effectiveness of endosonography versus surgical staging in potentially resectable lung cancer: a health economics analysis of the ASTER trial from a European perspective. Thorax 2014; 69: 679-681.

8 Yasufuku K, Pierre A, Darling G, et al. A prospective controlled trial of endobronchial ultrasound-guided transbronchial needle aspiration compared with mediastinoscopy for mediastinal lymph node staging of lung cancer. J Thorac Cardiovasc Surg 2011; 142: 1393-1400.

9 Czarnecka-Kujawa K, Yasufuku K. The role of endobronchial ultrasound versus mediastinoscopy for non-small cell lung cancer. J Thorac Dis 2017; 9: S83-S97.

10 Silvestri GA, Gonzalez AV, Jantz MA, et al. Methods for staging non-small cell lung cancer - Diagnosis and Management of Lung Cancer, 3rd edn: American College of Chest Physicians Evidence-Based Clinical Practice Guidelines. Chest 2013; 143: 5 Suppl, e211S-e250S.

11 Wahidi MM, Herth F, Yasufuku K, et al. Technical aspects of endobronchial ultrasound-guided transbronchial needle aspiration: CHEST guideline and expert panel report. Chest 2016; 149: 816-835.

12 Munoz ML, Lechtzin N, Li QK, et al. Bronchoscopy with endobronchial ultrasound guided transbronchial needle aspiration vs. transthoracic needle aspiration in lung cancer diagnosis and staging. J Thorac Dis 2017; 9 ; $2178-2185$. 
13 Chandra S, Nehra M, Agarwal D, et al. Diagnostic accuracy of endobronchial ultrasound-guided transbronchial needle biopsy in mediastinal lymphadenopathy: a systematic review and meta-analysis. Respir Care 2012; 57: 384-391.

14 Naur TMH, Konge L, Clementsen PF. Endobronchial ultrasound-guided transbronchial needle aspiration for staging of patients with non-small cell lung cancer without mediastinal involvement at positron emission tomography-computed tomography. Respiration 2017; 94: 279-284.

15 Van Schil P, van der Schoot J, Poniewierski J, et al. Remediastinoscopy after neoadjuvant therapy for non-small cell lung cancer. Lung Cancer 2002; 37: 281-285.

16 Mateu-Navarro M, Rami-Porta R, Bastus-Piulats R, et al. Remediastinoscopy after induction chemotherapy in non-small cell lung cancer. Ann Thorac Surg 2000; 70: 391-395.

17 Geake J, Hammerschlag G, Nguyen P, et al. Utility of EBUS-TBNA for diagnosis of mediastinal tuberculous lymphadenitis: a multicentre Australian experience. J Thorac Dis 2015; 7: 439-448.

18 Dziedzic DA, Peryt A, Orlowski T. The role of EBUS-TBNA and standard bronchoscopic modalities in the diagnosis of sarcoidosis. Clin Respir J 2017; 11: 58-63.

19 Li P, Zheng W, Zhao L. Convex probe endobronchial ultrasound: applications beyond conventional indications. J Thorac Dis 2015; 7: E289-E297.

20 Vilmann P, Frost Clementsen P, Colella S, et al. Combined endobronchial and esophageal endosonography for the diagnosis and staging of lung cancer: European Society of Gastrointestinal Endoscopy (ESGE) guideline, in cooperation with the European Respiratory Society (ERS) and the European Society of Thoracic Surgeons (ESTS). Eur J Cardiothorac Surg 2015; 48: 1-15.

21 Oki M, Saka H, Kitagawa C, et al. Endobronchial ultrasound-guided transbronchial biopsy using novel thin bronchoscope for diagnosis of peripheral pulmonary lesions. J Thorac Oncol 2009; 4: 1274-1277.

22 Jalil BA, Yasufuku K, Khan AM. Uses, limitations, and complications of endobronchial ultrasound. Proc (Bayl Univ Med Cent) 2015; 28: 325-330.

23 Balamugesh T, Herth FJ. Endobronchial ultrasound: a new innovation in bronchoscopy. Lung India 2009; 26: $17-21$.

24 Gierada D, Pinsky P, Duan F, et al. Interval lung cancer after a negative CT screening examination: CT findings and outcomes in national lung screening trial participants. Eur Radiol 2017; 27: 3249-3256.

25 Walter JE, Heuvelmans MA, Yousaf-Khan U, et al. New subsolid pulmonary nodules in lung cancer screening: The NELSON trial. J Thorac Oncol 2018; 13: 1410-1414.

26 Chen A, Chenna P, Loiselle A, et al. Radial probe endobronchial ultrasound for peripheral pulmonary lesions. A 5-year institutional experience. Ann Am Thorac Soc 2014; 11: 578-582.

27 Gomez M, Silvestri GA. Endobronchial ultrasound for the diagnosis and staging of lung cancer. Proc Am Thorac Soc 2009; 6: 180-186.

28 Kurimoto N, Miyazawa T, Okimasa S, et al. Endobronchial ultrasonography using a guide sheath increases the ability to diagnose peripheral pulmonary lesions endoscopically. Chest 2004; 126: 959-965.

29 Hürter T, Hanrath P. Endobronchial sonography: feasibility and preliminary results. Thorax 1992; 47: 565-567.

30 Yasufuku K. Current clinical applications of endobronchial ultrasound. Expert Rev Respir Med 2010; 4: 491-498.

31 Steinfort DP, Khor YH, Manser RL, et al. Radial probe endobronchial ultrasound for the diagnosis of peripheral lung cancer: systematic review and meta-analysis. Eur Respir J 2011; 37: 902-910.

32 Minezawa T, Okamura T, Yatsuya $\mathrm{H}$, et al. Bronchus sign on thin-section computed tomography is a powerful predictive factor for successful transbronchial biopsy using endobronchial ultrasound with a guide sheath for small peripheral lung lesions: a retrospective observational study. BMC Med Imaging 2015; 15: 21.

33 Durakovic A, Andersen H, Christiansen A, et al. Retrospective analysis of radial EBUS outcome for the diagnosis of peripheral pulmonary lesion: sensitivity and complications. Eur Clin Respir J 2015; 2: 28947.

34 Tanner NT, Yarmus L, Chen A, et al. Standard bronchoscopy with fluoroscopy $v s$ thin bronchoscopy and radial endobronchial ultrasound for biopsy of pulmonary lesions: a multicenter, prospective, randomized trial. Chest 2018; 154: 1035-1043.

35 Yarmus LB, Mallow C, Pastis N, et al. First-in-human use of a hybrid real-time ultrasound-guided fine-needle acquisition system for peripheral pulmonary lesions: a multicenter pilot study. Respiration 2019; 98: 527-533.

36 Vining DJ, Liu K, Choplin RH, et al. Virtual bronchoscopy: relationships of virtual reality endobronchial simulations to actual bronchoscopic findings. Chest 1996; 109: 549-553.

37 Seemann MD, Schaefer JF, Englmeier KH. Virtual positron emission tomography/computed tomography-bronchoscopy: possibilities, advantages and limitations of clinical application. Eur Radiol 2007; 17: 709-715.

38 Quon A, Napel S, Beaulieu CF, et al. "Flying through" and "flying around" a PET/CT scan: pilot study and development of 3D integrated 18F-FDG PET/CT for virtual bronchoscopy and colonoscopy. J Nucl Med 2006; 47: 1081-1087.

39 McLennan G, Namati E, Ganatra J, et al. Virtual bronchoscopy. Imaging Decis MRI 2007; 11: 10.

40 Pickering EM, Kalchiem-Dekel O, Sachdeva A. Electromagnetic navigation bronchoscopy: a comprehensive review. AME Med J 2018; 3: 117.

41 Rivera MP, Mehta AC, Wahidi MM. Establishing the diagnosis of lung cancer - Diagnosis and Management of Lung Cancer, 3rd edn: American College of Chest Physicians Evidence-Based Clinical Practice Guidelines. Chest 2013; 143: 5 Suppl, e142S-e165S.

42 Eberhardt R, Kahn N, Gompelmann D, et al. LungPoint: a new approach to peripheral lesions. J Thorac Oncol 2010; 5: 1559-1563.

43 DiBardino DM, Yarmus LB, Semaan RW. Transthoracic needle biopsy of the lung. J Thorac Dis 2015; 7: S304-S316.

44 Yarmus LB, Arias S, Feller-Kopman D, et al. Electromagnetic navigation transthoracic needle aspiration for the diagnosis of pulmonary nodules: a safety and feasibility pilot study. J Thorac Dis 2016; 8: 186-194.

45 Thiboutot J, Lee HJ, Silvestri GA, et al. Study design and rationale: a multicenter, prospective trial of electromagnetic bronchoscopic and electromagnetic transthoracic navigational approaches for the biopsy of peripheral pulmonary nodules. Contemp Clin Trials 2018; 71: 88-95. 

results of the AQuIRE registry. Am I Respir Crit Care Med 2016; 193: 68-77. Pritchett MA, Schampaert S, De Groot JAH, et al. Cone-beam CT with augmented fluoroscopy combined with electromagnetic navigation bronchoscopy for biopsy of pulmonary nodules. J Bronchol Intervent Pulmonol 2018; 25: $274-282$

48 Gould MK, Donington J, Lynch WR, et al. Evaluation of individuals with pulmonary nodules: when is it lung cancer? Diagnosis and Management of Lung Cancer, 3rd edn: American College of Chest Physicians Evidence-Based Clinical Practice Guidelines. Chest 2013; 143: 5 Suppl, e93S-e120S.

49 De Ruiter Q, Fontana J, Schampaert S, et al. Translation of a novel 3D navigation augmented fluoroscopy approach for endobronchial procedures based on CBCT. Chest 2018; 154: 1129A.

50 Krimsky WS, Pritchett MA, Lau KKW. Towards an optimization of bronchoscopic approaches to the diagnosis and treatment of the pulmonary nodules: a review. J Thorac Dis 2018; 10: S1637-S1644.

51 Rojas-Solano JR, Ugalde-Gamboa L, Machuzak M. Robotic bronchoscopy for diagnosis of suspected lung cancer: a feasibility study. J Bronchology Interv Pulmonol 2018; 25: 168-175.

52 Fielding D, Bashirzadeh F, Son JH, et al. First human use of a new robotic-assisted navigation system for small peripheral pulmonary nodules demonstrates good safety profile and high diagnostic yield. Chest 2017; 152 : A858.

53 Fielding DK, Bashirzadeh F, Son J, et al. First human use of a new robotic-assisted fiber optic sensing navigation system for small peripheral pulmonary nodules. Respiration 2019; 98: 142-150.

54 Yarmus L, Hopkins Hospital FJ, Wahidi M, et al. The precision-1 study: a prospective single-blinded randomized comparative study of three guided bronchoscopic approaches for investigating pulmonary nodules. Chest 2019; 156: A2256.

55 Romagnoli M, Colby TV, Berthet J, et al. Poor concordance between sequential transbronchial lung cryobiopsy and surgical lung biopsy in the diagnosis of diffuse interstitial lung diseases. Am J Respir Crit Care Med 2019; 199: $1249-1256$.

56 Troy LK, Grainge C, Corte TJ, et al. Diagnostic accuracy of transbronchial lung cryobiopsy for interstitial lung disease diagnosis (COLDICE): a prospective, comparative study. Lancet Respir Med 2019; 8: 171-181.

57 Hetzel J, Maldonado F, Ravaglia C, et al. Transbronchial cryobiopsies for the diagnosis of diffuse parenchymal lung diseases: expert statement from the Cryobiopsy Working Group on Safety and Utility and a call for standardization of the procedure. Respiration 2018; 95: 188-200.

58 Thiberville L, Salaün M, Lachkar S, et al. Confocal fluorescence endomicroscopy of the human airways. Proc Am Thorac Soc 2009; 6: 444-449.

59 Takemura M, Kurimoto N, Hoshikawa M, et al. Probe-based confocal laser endomicroscopy for rapid on-site evaluation of transbronchial biopsy specimens. Thorac Cancer 2019; 10: 1441-1447.

60 Hassan T, Thiberville L, Hermant C, et al. Assessing the feasibility of confocal laser endomicroscopy in solitary pulmonary nodules for different part of the lungs, using either 0.6 or $1.4 \mathrm{~mm}$ probes. PLoS One 2017; 12 : 1 .

61 Hassan T, Piton N, Lachkar S, et al. A novel method for in vivo imaging of solitary lung nodules using navigational bronchoscopy and confocal laser microendoscopy. Lung 2015; 193: 773-778.

62 Lin C, Yu K, Chang L, et al. Differentiating malignant and benign lymph nodes using endobronchial ultrasound elastography. J Formos Med Assoc 2019; 118: 436-443.

63 Izumo T. EBUS elastography for thoracic lesions. Ultrasound Med Biol 2017; 43: S31.

64 Michel RG, Kinasewitz GT, Fung KM, et al. Optical coherence tomography as an adjunct to flexible bronchoscopy in the diagnosis of lung cancer: a pilot study. Chest 2010; 138: 984-988.

65 Tsuboi M, Hayashi A, Ikeda N, et al. Optical coherence tomography in the diagnosis of bronchial lesions. Lung Cancer 2005; 49: 387-394.

66 Tan KM, Shishkov M, Chee A, et al. Flexible transbronchial optical frequency domain imaging smart needle for biopsy guidance. Biomed Opt Express 2012; 3: 1947-1954.

67 Kuo WC, Kim J, Shemonski ND, et al. Real-time three-dimensional optical coherence tomography image-guided core-needle biopsy system. Biomed Opt Express 2012; 3: 1149-1161.

68 Hariri LP, Villiger M, Applegate MB, et al. Seeing beyond the bronchoscope to increase the diagnostic yield of bronchoscopic biopsy. Am J Respir Crit Care Med 2013; 187: 125-129. 